\title{
Simulating sympathetic cooling of atomic mixtures in nonlinear traps
}

\author{
Francisco Jauffred, ${ }^{1}$ Roberto Onofrio, ${ }^{2,3}$ and Bala Sundaram ${ }^{1}$ \\ ${ }^{1}$ Department of Physics, University of Massachusetts, Boston, MA 02125, USA \\ ${ }^{2}$ Dipartimento di Fisica e Astronomia "Galileo Galilei", Università di Padova, Via Marzolo 8, Padova 35131, Italy \\ ${ }^{3}$ Department of Physics and Astronomy, Dartmouth College, 6127 Wilder Laboratory, Hanover, NH 03755, USA
}

\begin{abstract}
We discuss the dynamics of sympathetic cooling of atomic mixtures in realistic, nonlinear trapping potentials using a microscopic effective model developed earlier for harmonic traps. We contrast the effectiveness of different atomic traps, such as Ioffe-Pritchard magnetic traps and optical dipole traps, and the role their intrinsic nonlinearity plays in speeding up or slowing down thermalization between the two atomic species. This discussion includes cases of configurations with lower effective dimensionality. From a more theoretical standpoint, our results provide the first exploration of a generalized Caldeira-Leggett model with nonlinearities both in the trapping potential as well as in the interspecies interactions, and no limitations on their coupling strength.
\end{abstract}

\section{INTRODUCTION}

Recent progress in cooling fermions to the deep quantum degenerate regime has allowed the exploration of unique physics such as the crossover from the Bardeen-Cooper-Schrieffer (BCS) superfluid regime of Cooper pairs to the Bose-Einstein condensate (BEC) regime of tightly confined fermionic pairs [1, 2. This result is a major breakthrough in atomic physics with many interdisciplinary implications, from high- $\mathrm{T}_{c}$ superconductivity to neutron stars 35 . The continued exploration of even deeper Fermi regimes will expose quantum phase transitions and superconducting phases beyond the standard BCS scenario [6, 7. It is therefore not surprising that a considerable number of recent experiments have focused on trapping and cooling ever larger number of fermions through a variety of techniques [811]. The most successful and widespread technique is the sympathetic cooling of fermions by using a large Bose gas reservoir. However, the realization of novel behaviour alluded to earlier depends on the ability to optimize sympathetic cooling of Bose-Fermi mixtures. This effort includes attempts to maximize the number of available bosons [12, 13, where a crucial stage is the transfer from a magneto-optical trap into the conservative potential provided by either a magnetic trap or an optical dipole trap. Currently, loading efficiencies are around $10 \%$ providing considerable room for improvement. Increased numbers leading to larger bosonic clouds will also be more efficient in driving the fermionic counterpart into a quantum degenerate regime. Besides these practical considerations, Bose-Fermi mixtures are important prototypical systems, in which fundamental questions of thermalization, mixing, phase separation of general interest, especially in chemical physics, can be addressed in a pristine and controllable environment 14 .

Our earlier work [15] introduced an effective microscopic model to discuss thermalization dynamics between two atomic species in the nondegenerate regime. A key ingredient was an interaction term intended to extend CaldeiraLeggett models [16 23] to traps confining a finite number of atoms and arbitrary inter-species interaction strength. The model was later generalized to the more realistic and richer three-dimensional setting [24. In both instances, the trapping potential used was the idealized harmonic one, though a number of nontrivial effects resulted from the nonlinear character of the interatomic interaction. These included scaling with respect to the number of atoms, remiscent of Kolmogorov scaling in fluid mixing, and a mode locking mechanism which occurs in the presence of number-asymmetry in the two species. However, harmonic trapping does not realistically describe the early stage of atomic transfer, more manifestly because it assumes an infinite energy depth. The absence of a finite energy scale and the fixed oscillation period ensures that the thermalization dynamics is invariant with respect to an arbitrary common rescaling of the temperatures of the two species. Realistic trapping potentials have instead finite trapping depths and consequent breaking of the energy scaling invariance, which implies that one needs to specify the thermalization dynamics for each set of initial temperatures of the gases.

In this paper, we focus on nonlinear traps, with special emphasis on Ioffe-Pritchard magnetic traps and optical dipole traps, and their impact on the dynamics of thermalization. For this study, realistic insights require dealing with the full three-dimensional case, as one-dimensional analyses are too restrictive especially in the case of a Ioffe-Pritchard trap. Effective lower dimensional dynamics can be realized by using parameters corresponding to anisotropic traps. The interplay between nonlinearities of the trapping potential and the interaction term, responsible for thermalization, is rather subtle and, while we illustrate this with concrete examples, we do not consider the analysis to be exhaustive. Anharmonicities in the trapping potential usually weaken the local trapping strength but also provide channels of mixing and ergodicity since the oscillation periods depend on the energy of the particles. Which feature will prevail depends on the concrete experimental setting and dedicated simulations should be considered for each specific case. 


\section{NONLINEAR TRAPS}

In order to gain quantitative insights, we focus on a magnetic trap (MT) of the Ioffe-Pritchard type, due to its simplicity and widespread use. In this case the magnetic field vector is expressed as [25, 26]

$$
\mathbf{B}=B_{0}\left(\begin{array}{l}
0 \\
0 \\
1
\end{array}\right)+B^{\prime}\left(\begin{array}{c}
x \\
-y \\
0
\end{array}\right)+\frac{B^{\prime \prime}}{2}\left(\begin{array}{c}
-x z \\
-y z \\
z^{2}-\frac{1}{2}\left(x^{2}+y^{2}\right)
\end{array}\right)
$$

which corresponds to an amplitude

$$
B(x, y, z)=\left[\left(B^{\prime}-\frac{B^{\prime \prime}}{2} z\right)^{2} x^{2}+\left(B^{\prime}+\frac{B^{\prime \prime}}{2} z\right)^{2} y^{2}+\left(B_{0}+\frac{B^{\prime \prime}}{2}\left(z^{2}-\frac{1}{2}\left(x^{2}+y^{2}\right)\right)\right)^{2}\right]^{1 / 2}
$$

where the associated potential energy $V_{\text {mag }}=-\mu \cdot \mathbf{B}$. The magnetic dipole force acts on the trappable atoms (with magnetic moment antiparallel to the magnetic field) along the three Cartesian components. The components of the force acting on a generic magnetic moment in a Ioffe-Pritchard trap can be written as

$$
\begin{aligned}
& F_{x}=-\mu \frac{\left(B^{\prime}-\frac{B^{\prime \prime} z}{2}\right)^{2}-\frac{1}{2}\left[B_{0}+\frac{B^{\prime \prime}}{2}\left[z^{2}-\frac{1}{2}\left(x^{2}+y^{2}\right)\right]\right] B^{\prime \prime}}{\left[\left(B^{\prime}-\frac{B^{\prime \prime} z}{2}\right)^{2} x^{2}+\left(B^{\prime}+\frac{B^{\prime \prime} z}{2}\right)^{2} y^{2}+\left[B_{0}+\frac{B^{\prime \prime}}{2}\left[z^{2}-\frac{1}{2}\left(x^{2}+y^{2}\right)\right]\right]^{2}\right]^{1 / 2}} x, \\
& F_{y}=-\mu \frac{\left(B^{\prime}+\frac{B^{\prime \prime} z}{2}\right)^{2}-\frac{1}{2}\left[B_{0}+\frac{B^{\prime \prime}}{2}\left[z^{2}-\frac{1}{2}\left(x^{2}+y^{2}\right)\right]\right] B^{\prime \prime}}{\left[\left(B^{\prime}-\frac{B^{\prime \prime} z}{2}\right)^{2} x^{2}+\left(B^{\prime}+\frac{B^{\prime \prime} z}{2}\right)^{2} y^{2}+\left[B_{0}+\frac{B^{\prime \prime}}{2}\left[z^{2}-\frac{1}{2}\left(x^{2}+y^{2}\right)\right]\right]^{2}\right]^{1 / 2}} y, \\
& F_{z}=-\mu B^{\prime \prime} \frac{-\frac{1}{2}\left(B^{\prime}-\frac{B^{\prime \prime} z}{2}\right) x^{2}+\frac{1}{2}\left(B^{\prime}+\frac{B^{\prime \prime} z}{2}\right) y^{2}+\left[B_{0}+\frac{B^{\prime \prime}}{2}\left[z^{2}-\frac{1}{2}\left(x^{2}+y^{2}\right)\right]\right]}{\left[\left(B^{\prime}-\frac{B^{\prime \prime} z}{2}\right)^{2} x^{2}+\left(B^{\prime}+\frac{B^{\prime \prime} z}{2}\right)^{2} y^{2}+\left[B_{0}+\frac{B^{\prime \prime}}{2}\left[z^{2}-\frac{1}{2}\left(x^{2}+y^{2}\right)\right]\right]^{2}\right]} .
\end{aligned}
$$

As clear from the expression for the magnetic field, the potential is not fully symmetric around the $z$-axis. Exchange in $x$ and $y$ is affected by a relative minus sign in the gradient term (necessary to ensure a divergenceless magnetic

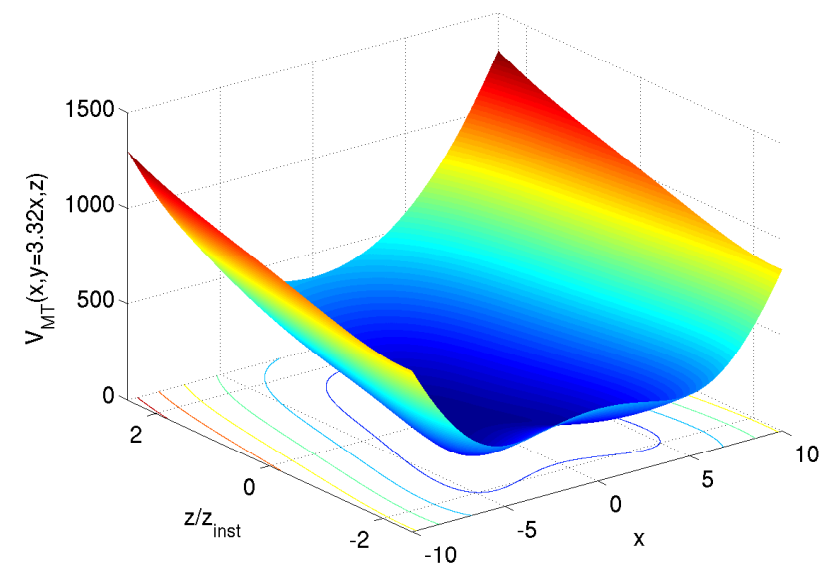

FIG. 1: Illustration of spatially asymmetric features of the magnetic trap potential. An arbitrary slice in the $(x, y)$ plane is shown, with the $z$ coordinate expressed in terms of the instability location $z_{\text {inst }}$. Instabilities in both radial and axial directions result in local potential minima which can retain atoms away from the center of the trap. All quantities are expressed in arbitrary units throughout the paper. 

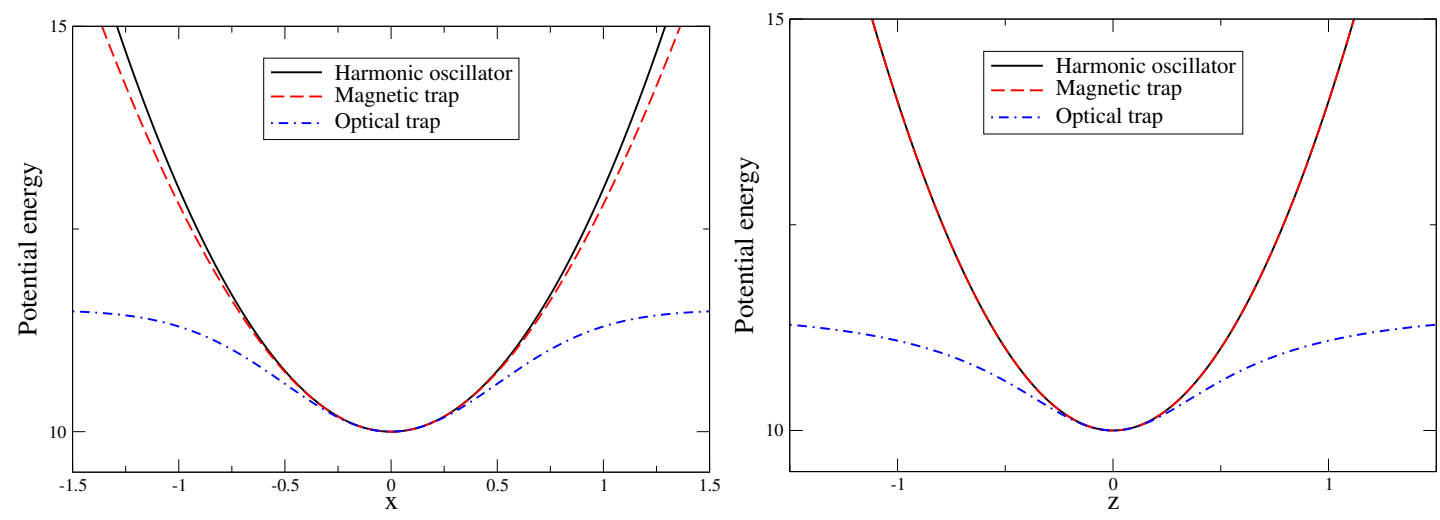

FIG. 2: Nonlinearities in realistic traps. The potential energy is shown versus the radial coordinate $x$ (left) and the axial coordinate $z$ (right) for the cases of a Ioffe-Pritchard magnetic trap (red, dashed curve), the ideal harmonic oscillator (black, continuous curve), and the optical dipole trap (blue, dot-dashed curve), all with the same small oscillation angular frequencies. The ideal harmonic oscillator and the optical potential have both been shifted by an amount $\mu B_{0}$ to coincide with the bottom of the magnetic trap potential. We have chosen $\mu=1, m=1, B_{0}=10, B^{\prime}=10$, and $B^{\prime \prime}=8$.

field), but in the small oscillation limit the trapping frequencies along the $x$ and $y$ axes are degenerate. The expressions specifying the frequencies in the three directions are $\omega_{x}^{2}(0)=\omega_{y}^{2}(0)=\mu\left(B^{\prime 2} / B_{0}-B^{\prime \prime} / 2\right) / m, \omega_{z}^{2}(0)=\mu B^{\prime \prime} / m$.

The more general, spatially-dependent, frequencies can be expressed in a relatively simple form by introducing two characteristic lengthscales of the trap, namely $\xi=\left(B_{0} / B^{\prime \prime}\right)^{1 / 2}$ and $\eta=B_{0} / B^{\prime}$. Then the expressions for the effective squared frequencies become

$$
\begin{aligned}
& \omega_{x}^{2}=\omega_{x}^{2}(0) \frac{1-z /\left[\left(\eta \xi^{2}\right)\left(1 / \eta^{2}-1 /\left(2 \xi^{2}\right)\right)\right]+\left(x^{2}+y^{2}\right) /\left[8 \xi^{4}\left(1 / \eta^{2}-1 /\left(2 \xi^{2}\right)\right)\right]}{\left\{\left[1+\left[z^{2}-\left(x^{2}+y^{2}\right) / 2\right] /\left(2 \xi^{2}\right)\right]^{2}+\left[1 / \eta-z /\left(2 \xi^{2}\right)\right]^{2} x^{2}+\left[1 / \eta+z /\left(2 \xi^{2}\right)\right]^{2} y^{2}\right\}^{1 / 2}}, \\
& \omega_{y}^{2}=\omega_{y}^{2}(0) \frac{1+z /\left[\left(\eta \xi^{2}\right)\left(1 / \eta^{2}-1 /\left(2 \xi^{2}\right)\right)\right]+\left(x^{2}+y^{2}\right) /\left[8 \xi^{4}\left(1 / \eta^{2}-1 /\left(2 \xi^{2}\right)\right)\right]}{\left\{\left[1+\left[z^{2}-\left(x^{2}+y^{2}\right) / 2\right] /\left(2 \xi^{2}\right)\right]^{2}+\left[1 / \eta-z /\left(2 \xi^{2}\right)\right]^{2} x^{2}+\left[1 / \eta+z /\left(2 \xi^{2}\right)\right]^{2} y^{2}\right\}^{1 / 2}}, \\
& \omega_{z}^{2}=\omega_{z}^{2}(0) \frac{1+\left[z^{2}-\left(x^{2}+y^{2}\right) / 2\right] /\left(2 \xi^{2}\right)-x^{2} /\left[2 z\left(1 / \eta-z /\left(2 \xi^{2}\right)\right)\right]+y^{2} /\left[2 z\left(1 / \eta+z /\left(2 \xi^{2}\right)\right)\right]}{\left\{\left[1+\left[z^{2}-\left(x^{2}+y^{2}\right) / 2\right] /\left(2 \xi^{2}\right)\right]^{2}+\left[1 / \eta-z /\left(2 \xi^{2}\right)\right]^{2} x^{2}+\left[1 / \eta+z /\left(2 \xi^{2}\right)\right]^{2} y^{2}\right\}^{1 / 2}} .
\end{aligned}
$$

Given the spatial complexity of the magnetic potential, a determination of the trap depth is rather involved, requiring numerical evaluation. There are three input parameters characterizing the magnetic field, bias field $B_{0}$, field gradient $B^{\prime}$, and field curvature $B^{\prime \prime}$, and three output parameters, trap depth, radial frequency, and axial frequency. As seen by inspecting Eq. (1), instabilities in atomic trapping occur for two reasons. First, the $B_{x}$ or $B_{y}$ components change sign, which happens at threshold values of the $z$ coordinate $z_{\text {inst }}= \pm 2 B^{\prime} / B^{\prime \prime}$, regardless of the values in the $(x, y)$ plane. Second, the $B_{z}$ component can also change sign, which occurs for all points $(x, y, z)$ such that $B_{0}+\left(B^{\prime \prime} / 2\right)\left[z^{2}-\frac{1}{2}\left(x^{2}+y^{2}\right)\right]=0$. This can be recast as a spatial condition

$$
x^{2}+y^{2}=2 z^{2}+4 B_{0} / B^{\prime \prime},
$$

i.e. a continuum of circles in the $(x, y)$ plane, with radii starting from a minimum value of $2 \sqrt{B_{0} / B^{\prime \prime}}$ at $z=0$, and increasing quadratically with $|z|$. Notice that the latter instability is dynamical, as it is conditional on achieving a well-defined value of $z$, while the former is fixed by the parameters of the trapping potential alone, a consideration which will play a role in interpreting simulation outcomes later on. The resulting potential depth will be, of course, the minimum value between the ones resulting from these two instability conditions. An indication of the presence of instabilities is seen in Fig. 1. where the magnetic potential is shown in $z$ and along a specific direction in the $x-y$ plane. These result in local valleys in the potential which can segregate more energetic atoms from the main clouds and affect interactions and, thus, thermalization. Note that what is shown is the magnitude of the potential which does not entirely reflect changes in the signs of the magnetic force at these instabilities. In other words, the antitrapping features of the potential are not seen. 
We now shift attention to the second type of nonlinear trap we consider, a single-beam optical dipole trap (ODT) with potential energy

$$
V_{\mathrm{opt}}(x, y, z)=V_{0}\left[1-\frac{\exp \left(\frac{-2\left(x^{2}+y^{2}\right)}{w_{0}^{2}\left(1+\left(z / z_{R}\right)^{2}\right)}\right)}{1+\left(z / z_{R}\right)^{2}}\right] .
$$

The potential is uniquely defined by the parameter $V_{0}$, the waist $w_{0}$, and the Rayleigh range $z_{R}=\pi w_{0}^{2} / \lambda_{L}$, where $\lambda_{L}$ is the laser wavelength. The potential energy amplitude $V_{0}$ is linearly related to the laser power and coincides with the trap depth, i.e. the maximum energy required to escape from the trap. These three parameters unambigously define the three trapping frequencies for small oscillations around the trap minimum. Due to the cylindrical symmetry around the $z$ axis two frequencies coincide, and the explicit dipole force acting on the atoms is expressed, in terms of its Cartesian components $\left(F_{x}, F_{y}, F_{z}\right)$, as

$$
\begin{gathered}
F_{x}=-\frac{4 V_{0}}{w_{0}^{2}} \frac{\exp \left(\frac{-2\left(x^{2}+y^{2}\right)}{w_{0}^{2}\left(1+\left(z / z_{R}\right)^{2}\right)}\right)}{\left[1+\left(z / z_{R}\right)^{2}\right]^{2}} x, \\
F_{y}=-\frac{4 V_{0}}{w_{0}^{2}} \frac{\exp \left(\frac{-2\left(x^{2}+y^{2}\right)}{w_{0}^{2}\left(1+\left(z / z_{R}\right)^{2}\right)}\right)}{\left[1+\left(z / z_{R}\right)^{2}\right]^{2}} y, \\
F_{z}=-\frac{2 V_{0}}{z_{R}^{2}} \frac{\exp \left(\frac{-2\left(x^{2}+y^{2}\right)}{w_{0}^{2}\left(1+\left(z / z_{R}\right)^{2}\right)}\right)}{\left[1+\left(z / z_{R}\right)^{2}\right]^{2}}\left[1-\frac{2}{w_{0}^{2}} \frac{x^{2}+y^{2}}{1+\left(z / z_{R}\right)^{2}}\right] z .
\end{gathered}
$$

The small oscillation frequencies are given by $\omega_{x}^{2}(0)=\omega_{y}^{2}(0)=4 V_{0} /\left(m w_{0}^{2}\right), \omega_{z}^{2}(0)=2 V_{0} /\left(m z_{r}^{2}\right)$. The nonlinearities are factorized in Eqs. $7 \mathrm{a} \mid 7 \mathrm{~b} / 7 \mathrm{c})$, and therefore the local oscillation frequency experienced by the atoms at a generic position can be easily rewritten as the small oscillation frequencies multiplied by a spatially-dependent nonlinear factor. Figure 2 illustrates the large distance deviations of the two realistic trapping potentials from the idealized harmonic approximation with the same small oscillation frequency.

In general, the optical dipole trap potential deviates more from the ideal harmonic case at smaller distances than the magnetic trap potential. This also results in an overall change in curvature which, as discussed in further detail in the next section, may be detrimental to thermalization dynamics. Experimental groups working on alloptical cooling to achieve degenerate quantum gases are well-aware of this drawback of optical dipole traps [27. In particular, evaporative cooling was found to become less efficient due to the simultaneous reduction of potential depth and curvature of the trap, the latter lowering significantly the elastic scattering rate necessary to repopulate the Boltzmann high-energy tail. Nevertheless, optical dipole traps are convenient especially as they allow for the independent use of tunable magnetic fields to control the scattering properties of the atoms. While this drawback has not prevented achieving Bose-Einstein condensation in the more complex configuration of two crossed beams [28], various techniques have been discussed to mitigate the progressive decrease in atomic density [29 33 .

In our context, additional issues with ODTs occur in the peripheral regions of the trap, critical in the early stage of evaporation and sympathetic cooling. From the expression given in Eq. $7 \mathrm{c}$, it is clear that the force in the $z$-direction can reverse sign. This occurs at thresholds given by

$$
x^{2}+y^{2}=\frac{w_{0}^{2}}{2}\left[1+\left(z / z_{R}\right)^{2}\right],
$$

which are, analogously to Eq. (5), concentric circles starting at a radius $w_{0} / \sqrt{2}$. This dynamical instability in the radial direction depends on the parameters of the trapping potential. As discussed in the context of the numerical results, this dependence suggests a mechanism for pushing this instability further out from the trap center which could assist thermalization.

\section{NUMERICAL RESULTS}

Within this framework, we have run numerical simulations using the Hamiltonian

$$
H_{\text {tot }}=\sum_{m=1}^{N_{p}}\left(\frac{P_{m}^{2}}{2 M}+V\left(Q_{m}\right)\right)+\sum_{n=1}^{N_{b}}\left(\frac{p_{n}^{2}}{2 m}+V\left(q_{n}\right)\right)+\gamma_{E} \sum_{m=1}^{N_{p}} \sum_{n=1}^{N_{b}} \exp \left[-\frac{\left(q_{n}-Q_{m}\right)^{2}}{\lambda^{2}}\right],
$$



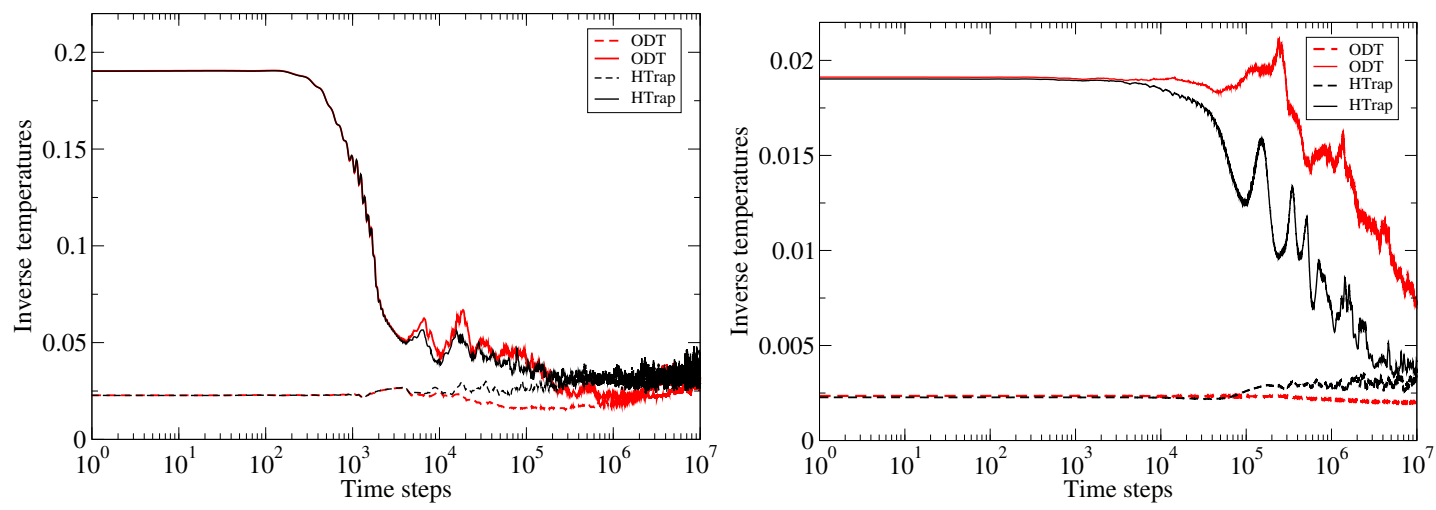

FIG. 3: Comparison between the thermalization dynamics for trapping potentials corresponding to an ideal harmonic confinement and to a realistic optical dipole trap due to a Gaussian-shaped laser beam. The common parameters are $N_{b}=N_{t}=100, \gamma_{E}=-1, \lambda=10$. In (a), the initial inverse temperatures are $\beta_{p}=0.02$ and $\beta_{b}=0.2$, while in (b) they are both lower by a factor of 10 , i.e. $\beta_{p}=0.002$ and $\beta_{b}=0.02$. The parameters of the equivalent Ioffe-Pritchard trap are $B_{0}=20, B^{\prime}=10, B^{\prime \prime}=0.01$, corresponding to a quasi-one dimensional trap with small oscillation frequencies $\omega_{x}=\omega_{y}=2.235$ and $\omega_{z}=0.1$ for both bath and test particles. Whereas in the low-temperature case the thermalization plots are nearly indistinguishable, at the higher temperatures the nonlinearity of the optical dipole trap results in a thermalization timescale almost one order of magnitude larger than the corresponding harmonic oscillator situation.

where $\left(q_{n}-Q_{m}\right)^{2}=\left(x_{n}-X_{m}\right)^{2}+\left(y_{n}-Y_{m}\right)^{2}+\left(z_{n}-Z_{m}\right)^{2} . q_{n}=\left(x_{n}, y_{n}, z_{n}\right)$ and $Q_{m}=\left(X_{m}, Y_{m}, Z_{m}\right)$ are the Cartesian coordinates of the particles in the two species, and $V$ is the harmonic, ODT or MT potential. The particle motion is completely described by vectors of dimensions $6 N_{b}$, i.e. $\left(q_{n}, p_{n}\right)$ and $6 N_{p}$, i.e. $\left(Q_{m}, P_{m}\right)$, respectively, where $N_{b}$ is the number of the coolant atoms (constituting the 'bath') and $N_{p}$ the number of particles of the cooled species. The interaction potential is the one we introduced earlier [15] and is characterized by a range $\lambda$, and a strength $\gamma_{E}$. Larger interaction strengths $\gamma_{E}$ obviously increase the thermalization speed. Up to a point, this occurs for larger values of $\lambda$ as well. Overall, the interaction term is genuinely local and nonlinear, but recovers the extended and linear case of the original Caldeira-Leggett model in the $\lambda \rightarrow \infty$ limit, for which thermalization of single-frequency baths is strongly inhibited [34].

We start from atomic clouds at different temperatures, assuming that they are initially prepared as if at equilibrium in a purely harmonic potential. The initial conditions for the simulations are drawn from these thermal distributions, a starting configuration which allows for a fair comparison of all the explored traps. This is consistent with the usual experimental protocol of precooling initial atomic clouds in a magneto-optical trap and then suddenly transferring them into the conservative trap. As detailed in [24, the inverse temperature is computed at each time step in terms of the variance in the total energy of species, $\sigma_{E}^{2}=\left\langle E^{2}\right\rangle-\langle E\rangle^{2}$, using the relationship $\beta=\sqrt{D} / \sigma_{E}$ valid for a Boltzmann energy distribution of a D-dimensional system. The results shown are for a single realization of the numerical experiment, which provides information on the shot-to-shot fluctuations, influenced by the finite number of atoms, which would be lost on averaging over several independent runs. Due to the relatively more complicated expressions, it is easier to choose the input parameters for the magnetic trap, and then derive parameters for the corresponding ideal harmonic trap and the optical dipole trap based on aligning the small oscillation trapping frequencies. We considered a wide range of parameters which can be broadly categorized in terms of the initial temperatures of the clouds and the relative values of the trapping frequencies in the different directions. At higher initial temperatures, the nonlinearities arising from the trapping potential are more relevant than at lower values and this serves to distinguish thermalization scenarios. The aspect ratio of the trapping frequencies determined by $\omega_{x} / \omega_{z}$, assuming cylindrical symmetry in keeping with experimental situations, changes the effective dimensionality of the particle dynamics with associated consequences for the thermalization dynamics.

Since the optical dipole trap differs significantly from the ideal case as shown in Figure 2 , we begin with a comparative analysis in the simplest case of quasi-one-dimensional dynamics, shown in Figure 3. The two panels (a) and (b) correspond to low and high initial cloud temperatures, respectively. As expected, at low temperatures, there is very little to differentiate between the two traps whereas, at higher temperatures, the harmonic trap is clearly more efficient in terms of thermalization, with the temperatures in the optical dipole trap case lagging behind the ones of the ideal case. In the case of the harmonic potential, notice also the distinct thermalization curves for the two temperature values. This is due to the nonlinear and nonperturbative nature of the interaction, for the large values of $\gamma_{E}$ and $\lambda$ chosen, indicating that a scaling property with temperature is not satisfied.

Next, we include the effect of dimensionality and the case of the magnetic trap. Figures 4 and 5 show results 
$1 \mathrm{D}$

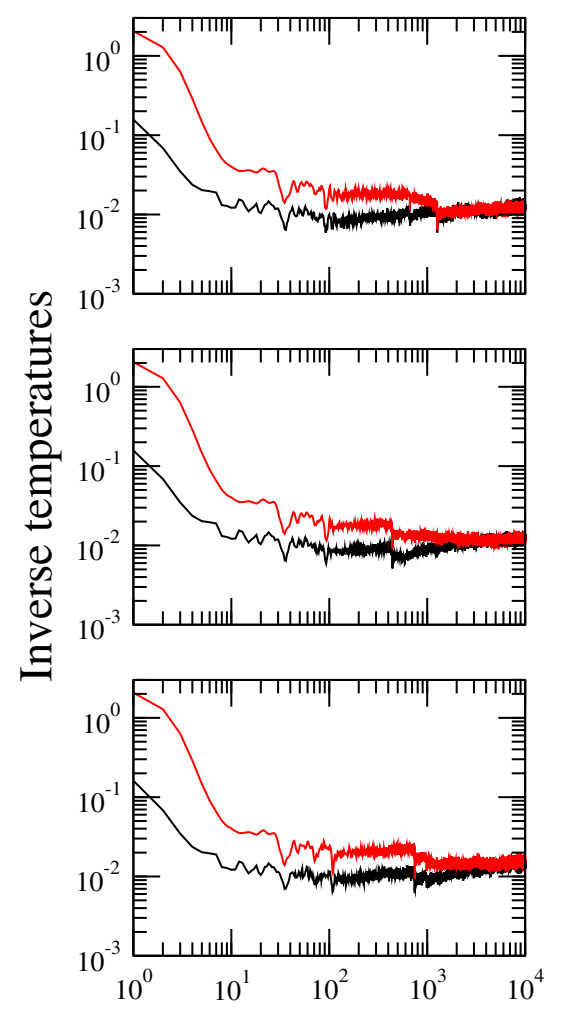

$2 \mathrm{D}$
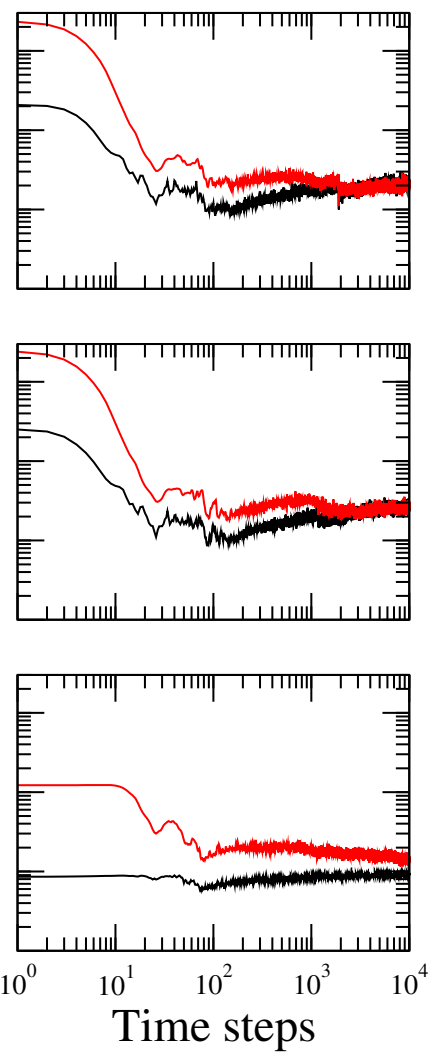

$3 \mathrm{D}$
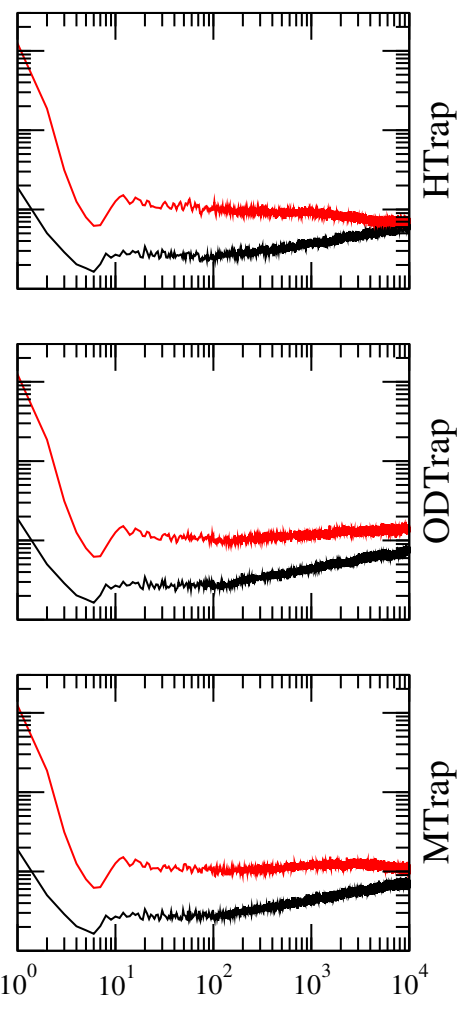

FIG. 4: Thermalization of mixtures in different traps and different effective dimensionality. The columns are relative to a comparison with a magnetic trap having $B_{0}=20, B^{\prime}=10$ in each case but $B^{\prime \prime}$ is changed such that the ratio of small oscillation frequencies result in different effective dimensional dynamics. The values for the three columns are $B^{\prime \prime}=0.1,9.8,3.3333$ respectively where the corresponding small oscillation frequencies $\left(\omega_{x}, \omega_{y}, \omega_{z}\right)$ are $(2.22, .2 .22,0.32),(0.33,0.33,3.13)$ and $(1.83,1.83,1.83)$ for either type of trap. The mixture is balanced, $N_{p}=N_{b}=500$, with the same interatomic interaction parameters, $\gamma_{E}=-14, \lambda=0.5$. The initial inverse temperatures are $\beta_{p}=0.2, \beta_{b}=2.0$. Note the exothermic nature of the interactions in all the cases shown, and the abrupt drop in inverse temperatures for the $2 \mathrm{D}$ magnetic trap case, as discussed in the text.

contrasting all three types of traps at low and high initial temperatures respectively, but for distinct frequency ratios. As seen from the figure captions, the triplet of magnetic field parameters $\left(B_{0}, B^{\prime}, B^{\prime \prime}\right)$ was chosen to stiffen the traps in one or more directions. The effective dimensionality of the dynamics is indicated at the top of each column. The low temperature results are clearly distinct from those at higher initial temperatures.

At lower initial temperatures (Fig. 4), the fact that for all effective dimensionalities the final convergent temperature is hotter than either of the initial ones is a clear indicator of the dominance of the interaction term, analogous to exothermic situations discussed earlier [15, 24]. For effective 1D dynamics, there is little to differentiate between the three trap configurations. In the 2D case, the MT shows signs of delayed convergence while the ODT closely follows the pure harmonic dynamics. Furthermore, this case shows an initial drop in inverse temperatures of both species which is not seen in any of the other cases. In the 3D situation, there are differences at late time while the early time dynamics are virtually identical. In particular, the ODT seems slightly less efficient with regard to thermalization, presumably due to the shape of the potential which differs significantly from the harmonic trap even at moderate distances from the center, as discussed in the context of Fig. 2.

The origins of the anomalous behavior observed in the MT 2D case arises from the instabilities, in $z$ and radial directions, alluded to earlier. For the stated magnetic field conditions, the threshold values for both instabilities is the least (closest to the origin) for the 2D situation. In fact, even at these low temperatures, substantial fractions of the initial conditions for both species lie beyond the threshold for the radial instability. These fractional values are 0.84 and 0.34 for target and bath species respectively. By contrast, the corresponding values in $1 \mathrm{D}$ and 3D are negligibly small for both instabilities. Also, the fractions beyond the threshold of the axial instability are 0.012 and 
$1 \mathrm{D}$

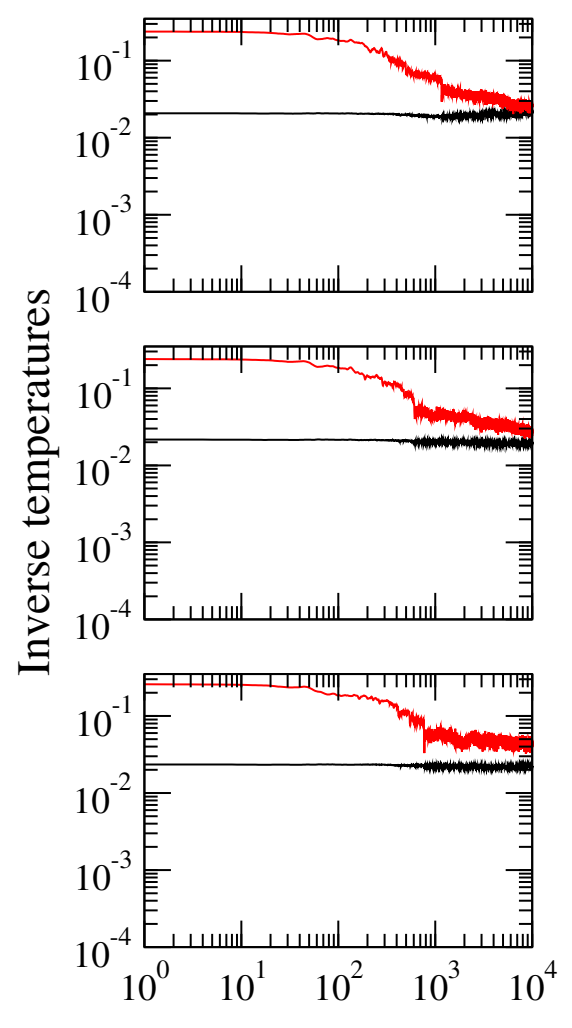

$2 \mathrm{D}$
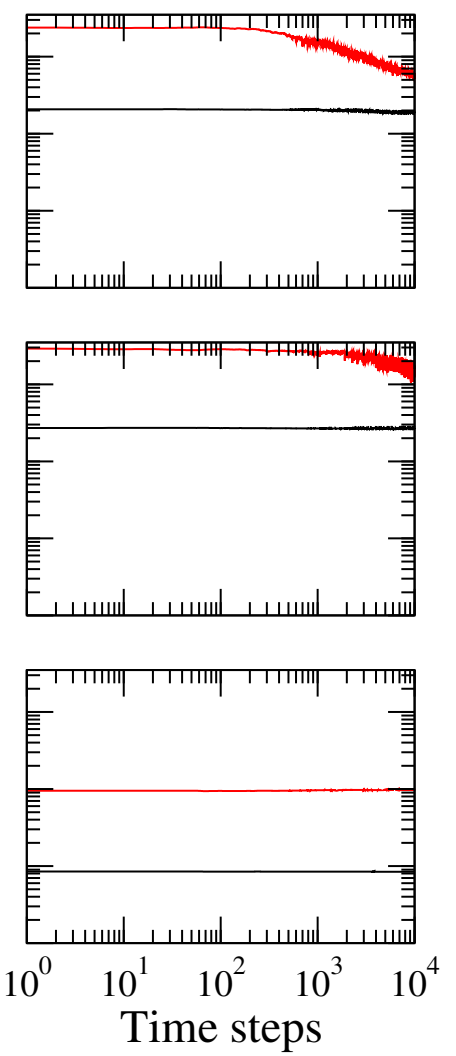

$3 \mathrm{D}$
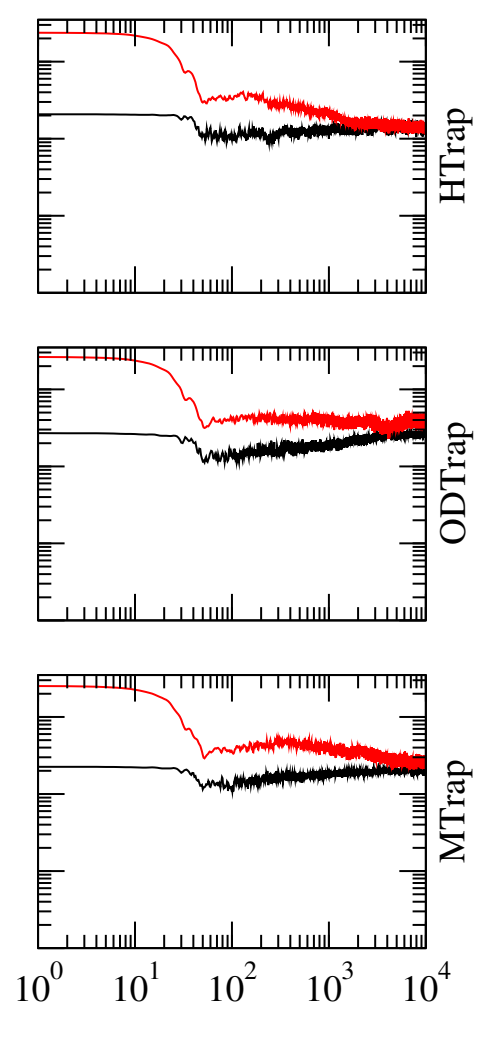

FIG. 5: Same conditions as in Figure 4 except that the initial conditions correspond to hotter temperatures. Here the starting inverse temperatures are $\beta_{p}=0.02, \beta_{b}=0.2$. Unlike at lower temperatures, the interaction is no longer manifestly exothermic, apart from the $2 \mathrm{D}$ magnetic trap case for which however thermalization is absent.

essentially zero for the $2 \mathrm{D}$ case. The radial instability appears to impact early time behavior while not dramatically altering thermalization at longer times.

For the higher initial temperatures shown in Fig. 5 even in the effective 1D situation, there are clear differences between the three types of traps with the harmonic one still being the most efficient. The converged temperature, in most of the cases shown, is trending towards a value between the two initial ones, or at least does not appear as exothermic as in the lower temperature case. For the harmonic trap, this is a consequence of the relative importance of the interaction energy as compared with the energies of the individual clouds, where the latter is dominant at these higher temperatures. Moreover, the spatially extended initial conditions at higher temperatures better explore the nonlinear features of the MT and ODT potentials. Two additional features are also worth noting.

First, the 3D cases thermalize better than the corresponding 1D instances. This is somewhat surprising as the expectation is that, in higher dimensions, the ability to avoid interactions should be enhanced. Our earlier results required higher values of $\gamma_{E}$ for higher dimensions to achieve comparable thermalization timescales [24], so we anticipated the 1D case to be even more efficient. A possible interpretation of this outcome is the role played by trapping regions in which the particle motion is chaotic. Previous work has explored the role of chaos for evaporative cooling in magnetic traps, concluding that the effective dimensionality of the process changes from being fully three-dimensional to one-dimensional as one moves from chaotic to integrable trajectories [35.38. Further, studies of magnetic traps [39] and crossed optical dipole traps [40] confirm the presence of chaotic trajectories for the most energetic particles.

Second, the 2D situation is clearly the anomaly with respect to the $1 \mathrm{D}$ and $3 \mathrm{D}$ cases and the MT $2 \mathrm{D}$ case is the most distinct as there are no signs of thermalization whatsoever. Contrasted with the low temperature case, not only are higher fractions of initial conditions above the radial threshold (now 0.97 and 0.84 for particles and bath respectively) but a significant fraction of the particles (0.52) are also above the threshold for the axial instability. By comparison, these fractions in $1 \mathrm{D}$ are negligible, while the $3 \mathrm{D}$ values are no larger than 0.28 . As indicated earlier, we infer from this that large fractions beyond the radial instability affect the early time dynamics, with heating leading 

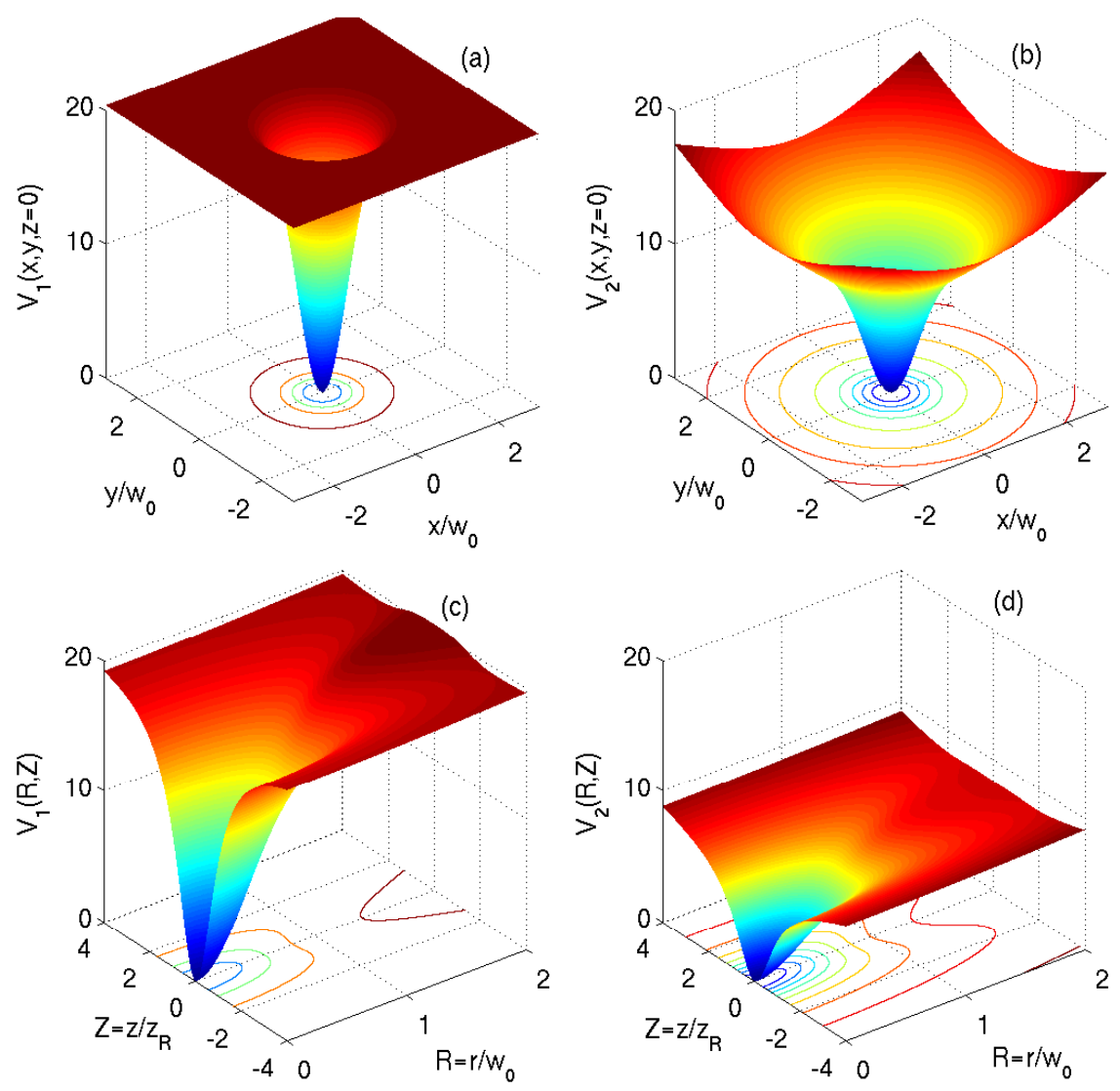

FIG. 6: Contrasting the usual ODT (a) with a more general double optical dipole trap (DODT) (b) for $z=0$, as discussed in the text, with $c_{0}=0.4$ and $c_{1}=0.6$. $(x, y)$ are expressed in terms of the beam waist. The presence of a second laser beam increases the retaining capability in the outer region of confinement in which the single optical dipole trap has a nearly flat potential. Panels (c) and (d) show the same two cases using the cylindrical symmetry, with $r$ relative to the waist and $z$ to the Rayleigh range $z_{R}$. The radial instability given by the condition Eq. [8] is clearly visible in (c) while the fact that the secondary beam has a waist which is five times the one of the primary beam pushes the instability further out in (d). Both waist and Rayleigh range of the auxiliary beam preserve the aspect ratio of the single trap.

to an abrupt drop in inverse temperatures immediately after the simulation starts. To reiterate, this is a consequence of the hotter atoms, associated with the higher initial temperatures, which are transferred from the precooling stage (typically from a magneto-optical trap) in which, as discussed above, harmonic trapping is assumed. Unlike the earlier situations, this effect is further compounded with the onset of the axial instability. We have contrasted trajectories, starting from initial conditions on either side of the instabilities, to gauge the impact of the two kinds of instabilities discussed. Similar runs were also used to extract Lyapunov exponents which clearly show the role of chaos in the dynamics above the threshold for instability.

The presence of threshold values for antitrapping regions, as in the MT case, or the very presence of trajectories quite far apart from the center of the trapping potential in general, strongly affect the thermalization efficiency. It is possible to mitigate this effect by adding an auxiliary potential having minimal effect near the center, while forcing atoms in the peripheral regions towards the center of the trap. This addition would also assist in the case of ODT where the softening of the potential, as seen in Fig. 2, can lead to shelving of fractions of the populations.

A secondary potential can be realized in the case of an optical dipole trap by splitting the original laser beam into two components, and sending the two beams with two different focal lengths on the center of the optical dipole trap. The laser beam corresponding to minimal beam waist is aimed at maintaining a strong confinement at the center, while the one with larger waist gives rise to a stiffer potential on the periphery. We consider two laser beams with waists $w_{0}$ and $w_{1}$ and wavelengths $\lambda_{L 0}$ and $\lambda_{L 1}$. The Rayleigh ranges are then $z_{0}=\pi w_{0}^{2} / \lambda_{L 0}$ and $z_{1}=\pi w_{1}^{2} / \lambda_{L 1}$. We start with the simplifying hypothesis that the laser is common to the two beams, so we have $\lambda_{L 0}=\lambda_{L 1}$. The generic 


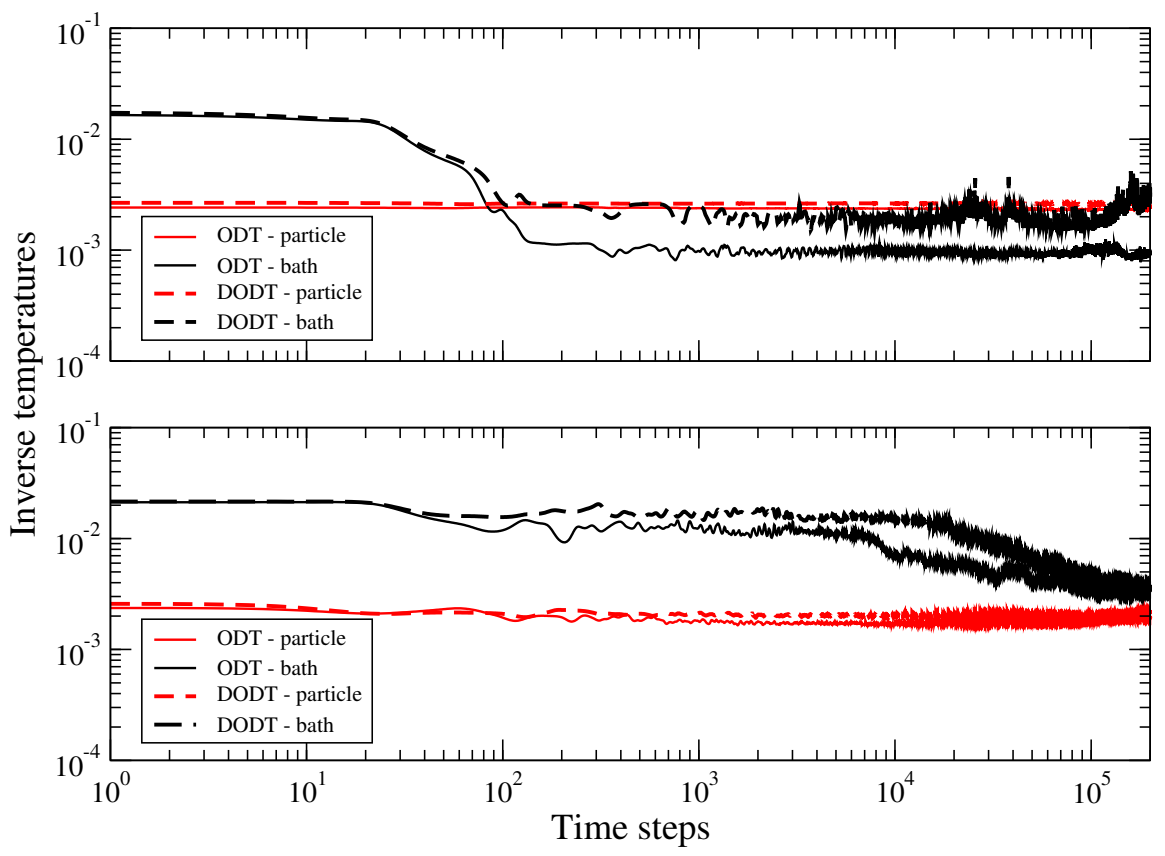

FIG. 7: Thermalization dynamics for optical dipole traps with and without an auxiliary laser beam with large waist, assisting the recapture of more energetic atoms. The top plot is relative to an unbalanced mixture in which $N_{b}=10, N_{p}=390$, the bottom plot for a balanced mixture $\left(N_{b}=N_{p}=200\right.$ ). Other parameters are common and equal to $\gamma_{E}=+14$ (corresponding to repulsive interspecies interactions enhancing the shelving effect), $\lambda=10, \beta_{p}=0.002, \beta_{b}=0.02, c_{0}=0.4, c_{1}=0.6$. The small oscillation frequencies for both ODT and DODT are $\left(\omega_{x}, \omega_{y}, \omega_{z}\right)=(6.95,6.95,1.83)$.

optical dipole potential is

$$
V_{\text {opt }}(x, y, z)=V_{0}\left[c_{0}+c_{1}-c_{0} \frac{\exp \left(\frac{-2\left(x^{2}+y^{2}\right)}{w_{0}^{2}\left(1+\left(z / z_{0}\right)^{2}\right)}\right)}{1+\left(z / z_{0}\right)^{2}}-c_{1} \frac{\exp \left(\frac{-2\left(x^{2}+y^{2}\right)}{w_{1}^{2}\left(1+\left(z / z_{1}\right)^{2}\right)}\right)}{1+\left(z / z_{1}\right)^{2}}\right] .
$$

The arbitrary offset of the potential is chosen in such a way that $V(0,0,0)=0$ and its asymptotic value at infinite distance from the origin is $V(|\infty|) / V_{0}=c_{0}+c_{1}$. In general, the expressions for the forces along the three directions can be readily obtained. These expressions, once linearized near the origin, give for the three Cartesian components of the force

$$
\begin{aligned}
& F_{x}=-4 V_{0}\left(\frac{c_{0}}{w_{0}^{2}}+\frac{c_{1}}{w_{1}^{2}}\right) x, \\
& F_{y}=-4 V_{0}\left(\frac{c_{0}}{w_{0}^{2}}+\frac{c_{1}}{w_{1}^{2}}\right) y, \\
& F_{z}=-2 V_{0}\left(\frac{c_{0}}{z_{0}^{2}}+\frac{c_{1}}{z_{1}^{2}}\right) z .
\end{aligned}
$$

Since we are interested in comparing the behaviour of the traps far from the center, we have chosen the parameters $c_{0}$ and $c_{1}$ and the laser waists such that the small oscillation frequencies for the ODT and double optical dipole trap (DODT) are the same. This more generalized potential is contrasted with the usual ODT in Fig. 6. Our choice implies more laser power in the DODT and hence a larger energy depth which is reflected in the continued growth of tails of the potential. Figure 7 contrasts the thermalization curves for the ODT and DODT cases in two situations; where the two species have equal numbers of particles and the other where there are substantionally fewer cooler bath particles. The initial temperatures of both clouds are chosen to be higher than the hottest cases analyzed so far in 
Fig. 5 to enhance the shelving effect and show the impact of the additional ODT. In the asymmetric particle case, the overall effect of the DODT is amplified with respect to the equal number mixture. We believe this is due to the fact that, for a single ODT, the small number of the cold species are quickly ejected to the flat peripheral regions of the potential due to their repulsive interactions with the considerably larger number of hot particles. Once this occurs, they have only very limited encounters with the other species, the condition needed for thermalization. The DODT provides the mechanism to reinject them towards the trap center resulting in renewed interactions. Also this would lead one to expect variability in the results with initial configuration of the colder particles. When the particle numbers are balanced, this mechanism is far less important and the thermalization timescales are comparable in the two cases. It would be interesting to explore the situation more relevant for cooling atoms, with a large cold reservoir of atoms trying to drive to lower temperatures a smaller number of hot atoms, though the particle numbers required to show this are beyond our current computational capabilities.

Analogous considerations can be applied to the case of a MT. The addition of a shallow, attractive optical potential should help return atoms more quickly to the minimum of the potential. However, the expression of the magnetic field in Eq. (1) contains only the leading term in a multipole expansion 25] which is not enough to capture the long-distance behavior of the trapped atoms. Detailed ab initio evaluations of the magnetic field are required to confirm that the lack of thermalization intrinsic to the high-temperature situation for the $2 \mathrm{D}$ case in Figure 5 is mitigated by the presence of a monotonically attractive optical potential term.

\section{CONCLUSIONS}

In conclusion, we have discussed the dynamics of thermalization of atomic mixtures for realistic atomic traps. The interplay between nonlinearities arising from the interatomic potential term, necessary to enforce thermalization, and the ones arising from nonlinear trapping, present in any realistic setup, has been emphasized. Comparison with the ideal harmonic trapping allows us to disentangle features arising from the nonlinearity of the realistic potentials. Thermalization is achieved in a large number of cases we have analyzed, with the notable exception of the magnetic trapping configuration in $2 \mathrm{D}$, where instabilities in the potential result in atoms moving away from regions of maximal interaction. This feature is clearly more detrimental at higher initial temperatures. We have also evidence for faster thermalization in 3D with respect to the corresponding 1D case, despite the inhibition of head-on collisions, an effect that may be influenced by dynamical chaos. The issue of whether the presence of chaotic behavior in nonlinear traps is advantageous to increasing the thermalization speed requires further exploration. However, our results do suggest that nonlinearities in atom traps may be turned into assets, as discussed also in Ref [4] with regard to precision measurements of the trap parameters on exploiting nonlinearities of the ODT. Simulations like the ones shown, appropriate for each specific experimental setup, will help identify parameters for which this effect can contribute significantly to the earlier stage of atomic cooling, especially during transfer of atoms from a magneto-optical trap into a conservative trap.

[1] M. W. Zwierlein, J. R. Abo-Shaeer, A. Schirotzek, C. H. Schunck, W. Ketterle, Vortices and superfluidity i a strongly interacting Fermi gas, Nature 435 (2005) 1047.

[2] W. Ketterle, M. W. Zwierlein, Making, probing, and understanding ultracold Fermi gases, Riv. Nuovo Cimento 31 (2008) 247.

[3] Q. Chen, J. Stajic, S. Tan, K. Levin, BCS-BEC crossover: From high temperature superconductors to ultracold superfluids, Phys. Rep. 412 (2005) 1.

[4] S. Giorgini, L. P. Pitaevskii, S. Stringari, Theory of ultracold atomic Fermi gases, Rev. Mod. Phys. 80 (2008) 1215.

[5] E. A. Ayryan, K. G. Petrosyan, A. H. Gevorgyan, N. Sh. Izmailian, Physics of cold atomic Fermi gases, arXiv:1703.00813 [cond-mat.quant-gas] (9 Mar 2017).

[6] D. C. McKay, B. DeMarco, Cooling in strongly correlated optical lattices: prospects and challenges, Rep. Progr. Phys. 74 (2011) 054401.

[7] R. Onofrio, Cooling and thermometry of atomic Fermi gases, Physics Uspekhi 59 (2016) 1129.

[8] P. M. Duarte, R. A. Hart, J. M. Hitchcock, T. A. Corcovolos, T.-L. Yang, A. Reed, R. G. Hulet, All-optical production of a lithium quantum gas using narrow-line laser cooling, Phys. Rev. A 84 (2011) 061406(R).

[9] D. C. McKay, D. Jervis, D. J. Fine, J. W. Simpson-Porco, G. J. A. Edge, J. H. Thywissen, Low-temperature high-density magnetic-optical trapping of potassium using the open $4 S \rightarrow 5 P$ transition at $405 \mathrm{~nm}$, Phys. Rev. A 84 (2011) 063420 .

[10] A. Burchianti, G. Valtolina, J. A. Serman, E. Pace, M. Pas, M. Inguscio, Efficient all-optical production of large ${ }^{6}$ Li quantum gases using $D_{1}$ gray-molasses cooling, Phys. Rev. A 90 (2014) 043408.

[11] Ch. Gross, H. C. J. Gan, K. Dieckmann, All-optical production and transport of a large ${ }^{6}$ Li quantum gas in a crossed optical dipole trap, Phys. Rev. A 93 (2016) 053424. 
[12] S. Chaudhuri, S. Roy, C. S. Unnikrishnan, Evaporative cooling of atoms to quantum degeneracy in an optical dipole trap, J. Phys. Conf. Ser. 80 (2007) 012036.

[13] K. Shibata, S. Yonekawa, S. Tojo, Loading of atoms into an optical trap with high initial phase-space density, arXiv:1611.08960v1 [cond-mat.quant-gas] 28 Nov 2016.

[14] C. Ufrecht, M. Meister, A. Roura, W. P. Schleich, Comprehensive classification for Bose-Fermi mixtures, arXiv:1702.06050v1 [cond-mat.quant-gas] 20 Feb 2017.

[15] R. Onofrio, B. Sundaram, Effective microscopic models for sympathetic cooling of atomic gases, Phys. Rev. A 92 (2015) 033422.

[16] V. B. Magalinskii, Dynamical model on the theory of the Brownian motion, Sov. Phys. JETP 9 (1959) 1381.

[17] P. Ullersma, An exactly solvable model for Brownian motion. 1. Derivation of Langevin equation, Physica 52 (1966) 27.

[18] P. Ullersma, An exactly solvable model for Brownian motion. 2. Derivation of Fokker-Planck equation and master equation, Physica 32 (1966) 56.

[19] P. Ullersma, An exactly solvable model for Brownian motion. 3. Motion of a heavy mass in a linear chain, Physica 32 (1966) 74 .

[20] P. Ullersma, An exactly solvable model for Brownian motion. 4. Susceptibility and Nyquist's theorem, Physica 32 (1966) 90.

[21] A. O. Caldeira, A. J. Leggett, Influence of dissipation on quantum tunneling in macroscopic systems, Phys. Rev. Lett. 46 (1981) 211.

[22] A. O. Caldeira, A. J. Leggett, Quantum tunnelling in a dissipative system, Ann. Phys. 149 (1983) 374.

[23] A. O. Caldeira, A. J. Leggett, Influence of damping on quantum interference: An exactly soluble model, Ann. Phys. 149 (1983) 374.

[24] F. Jauffred, R. Onofrio, B. Sundaram, Universal and anomalous behavior in the thermalization of strongly interacting harmonically trapped gas mixtures, J. Phys. B: At. Mol. Opt. Phys. 50 (2017) 135005.

[25] T. Bergeman, G. Erez, H. J. Metcalf, Magnetostatic trapping fields for neutral atoms, Phys. Rev. A 35 (1987) 1535.

[26] W. Ketterle, D. S. Durfee, D. M. Stamper-Kurn, Making, probing, and understanding Bose-Einstein condensates, in BoseEinstein condensation in atomic gases, Proceedings of the International School of Physics "Enrico Fermi", Course CXL (SIF, Bologna, 1999).

[27] C. S. Adams, H. J. Lee, N. Davidson, M. Kasevich, S. Chu, Evaporative cooling in a crossed dipole trap, Phys. Rev. Lett. 74 (1995) 3577.

[28] M. D. Barrett, J. A. Sauer, M. S. Chapman, All-optical formation of an atomic Bose-Einstein condensate, Phys. Rev. Lett. 87 (2001) 010404.

[29] G. Cennini, G. Ritt, C. Geckeler, M. Weitz, Bose-Einstein condensation in a $\mathrm{CO}_{2}$-laser optical dipole trap, Appl. Phys. B 77 (2003) 773.

[30] T. Kinoshita, T. Wenger, D. Weiss, All-optical Bose-Einstein condensation using a compressible crossed dipole trap, Phys. Rev. A 71 (2005) 011602(R).

[31] C.-L. Hung, X. Zhang, N. Gemelke, C. Chin, Accelerating evaporative cooling of atoms into Bose-Einstein condensation in optical traps, Phys. Rev. A 78 (2008) 011604(R).

[32] J.-F. Clément, J.-P. Brantut, M. Robert-de-Saint-Vincent, R. A. Nyman, A. Aspect, T. Bourdel, P. Bouyer, All-optical runaway evaporation to Bose-Einstein condensation, Phys. Rev. A 79 (2009) 061405(R).

[33] K. J. Arnold, M. D. Barrett, All-optical Bose-Einstein condensation in a $1.06 \mu \mathrm{m}$ dipole trap, Optics Communications 284 (2011) 3288.

[34] S. T. Smith, R. Onofrio, Thermalization in open classical systems with finite heat baths, Eur. Phys. J. B 61 (2008) 271.

[35] E. L. Surkov, J. T. M. Walraven, G. V. Shlyapnikov, Collisionless motion of neutral particles in magnetostatic traps, Phys. Rev. A 49 (1994) 4778.

[36] E. L. Surkov, J. T. M. Walraven, G. V. Shlyapnikov, Collisionless motion and evaporative cooling of atoms in magnetic traps, Phys. Rev. A 53 (1996) 3403.

[37] P. W. H. Pinkse, A. Mosk, M. Weidemüller, M. W. Reynolds, T. W. Hijmans, J. T. M. Walraven, One-dimensional evaporative cooling of magnetically trapped atomic hydrogen, Phys. Rev. A 57 (1998) 4747.

[38] V. G. O. Harms, D. Haubrich, H. Schadwinkel, F. Strauch, B. Ueberholtz, S. aus der Wiesche, D. Meschede, Magnetostatic traps for charged and neutral particles, Hyperfine Interactions 109 (1997) 281.

[39] J. P. Salas, M. Iñarrea, Regular and chaotic dynamics of a neutral atom in a magnetic trap, Eur. Phys. J. D 67 (2013) 248.

[40] R. González-Férez, M. Iñarrea, J. P. Salas, P. Schmelcher, Nonlinear dynamics of atoms in a crossed optical dipole trap, Phys. Rev. E 90 (2014) 062919.

[41] V. Makhalov, K. Martiyanov, T. Barmashova, A. Turlapov, Precision measurement of a trapping potential for an ultracold gas, Phys. Lett. A 379 (2015) 327. 